DOI: $10.1590 / 1089-6891 v 16 i 427635$

MEDICINA VETERINÁRIA

\title{
HEMOGLOBINA GLICADA E FRUTOSAMINA EM CÃES COM DIABETES MELLITUS
}

\section{GLYCATED HEMOGLOBIN AND FRUCTOSAMINE IN DOGS WITH DIABETES MELLITUS}

\author{
Olair Carlos Beltrame ${ }^{1^{*}}$ \\ Rosangela Locatelli-Dittrich ${ }^{1}$ \\ Luciane Maria Laskoski ${ }^{1}$ \\ Lia Fordiani Lenati Patricio ${ }^{1}$ \\ Nina da Cunha Medeiros ${ }^{1}$ \\ Marília Oliveira Koch ${ }^{1}$ \\ ${ }^{1}$ Departamento de Medicina Veterinária, Universidade Federal do Paraná, Curitiba, PR, Brasil. \\ *Autor para correspondência - beltrame@ufpr.br
}

\begin{abstract}
Resumo
O Diabetes mellitus (DM) ocorre com frequência em cães, sendo a glicemia o teste laboratorial de confirmação. O diagnóstico e monitoramento em pessoas são realizados pelas concentrações de hemoglobina glicada e frutosamina. O objetivo deste estudo foi diagnosticar a DM em 19 cães, com avaliação das concentrações da glicose sérica, hemoglobina glicada e frutosamina. Seis cães com DM e em tratamento com insulina foram acompanhados por um período de doze meses, com as mesmas avaliações hematológicas, até o óbito (três cães) ou controle glicêmico (três cães). Observouse aumento dos valores de glicose, hemoglobina glicada e frutosamina em todos os cães com DM, e valores de hemoglobina glicada e glicose superiores nos animais não sobreviventes, na última avaliação realizada. Estes resultados apontam para a importância da avaliação de hemoglobina glicada e frutosamina em cães com diabetes e para diagnóstico e controle da efetividade do tratamento.

Palavras-Chave: diagnóstico laboratorial; glicemia; insulina; prognóstico.
\end{abstract}

\begin{abstract}
Diabetes mellitus (DM) commonly occurs in dogs, and the laboratorial confirmation is carried out by glycemia test. The diagnosis and monitoring in humans is made by glycated hemoglobin and fructosamine concentrations. The objective of this study was to diagnose DM in 19 dogs, by evaluating seric glucose, glycated hemoglobin and fructosamine concentrations. Six dogs with DM and treated with insulin were assisted during a twelve-month period, by means of the same blood analysis, until the death (three dogs) or glycemic control (three dogs). Glucose, glycated hemoglobin and fructosamine increased in all dogs with DM, and dogs that did not survive presented higher glycated hemoglobin and seric glucose values than those that survived at the last evaluation. The results showed the importance of evaluating glycated hemoglobin and fructosamine in dogs with DM to diagnose and control treatment effectiveness.
\end{abstract}

Keywords: glycemia; insulin; laboratorial diagnostic; prognostic.

Recebido em: 10 dezembro 2013

Aceito em: 17 agosto 2015 


\section{Introdução}

A prevalência do Diabetes mellitus (DM) em cães vem aumentando consideravelmente nos últimos anos. O DM é uma endocrinopatia comum em cães e pode ser fatal se não for diagnosticada e adequadamente tratada. A deficiência de insulina que ocorre no DM é resultante da incapacidade das ilhotas pancreáticas em secretar insulina ou da sua ação deficiente nos tecidos ${ }^{(1)}$. $\mathrm{O}$ descontrole permanente acarreta uma série de complicações orgânicas, resultando em danos aos tecidos, perda de função e falência orgânica ${ }^{(2)}$. $\mathrm{O}$ diagnóstico baseia-se fundamentalmente no aumento da glicose plasmática em jejum ou pelo teste de tolerância à glicose ${ }^{(3)}$.

Os cães diabéticos do tipo 1 são insulino-dependentes e necessitam da mesma de forma contínua. Geralmente, são necessárias duas aplicações ao dia e o tratamento é bastante individualizado, sendo necessário o ajuste da dose ideal para cada paciente ${ }^{(4)}$.

A hemoglobina glicada e a frutosamina são muito utilizadas na medicina humana como exames padrão-ouro para diagnóstico, monitoramento e tratamento da DM, podendo ser utilizadas também para cães, desde que devidamente ajustadas com valores de referências para a espécie ${ }^{(5)}$. A dosagem da frutosamina e hemoglobina glicada são de extrema importância, por refletirem os níveis glicêmicos a curto e longo prazo e permiterem intervenção clínica em tempo útili ${ }^{(3,6)}$.

Dada a importância destes dois exames para pacientes diabéticos, objetivou-se avaliar cães com DM, em tratamento com insulina, por meio das dosagens de glicose sérica, hemoglobina glicada e frutosamina, e determinar valores de hemoglobina glicada e frutosamina relacionados com agravamento clínico e prognóstico desfavorável em cães com complicações resultantes da DM.

\section{Material e Métodos}

Este trabalho foi submetido e aprovado pela Comissão de Ética no Uso de Animais CEUA/SCA protocolo 048/2010. Foram utilizados 19 cães com diagnóstico de DM, de idades e raças variadas, provenientes do atendimento médico veterinário a animais de proprietários particulares, realizado no Hospital Veterinário, pelos residentes veterinários e professores da universidade. $\mathrm{O}$ diagnóstico de DM foi pautado em sinais clínicos como poliúria, glicosúria, polidipsia, polifagia e dosagem de glicose sérica superior a $110 \mathrm{mg} / \mathrm{dL}$. Posteriormente à primeira avaliação, os animais foram tratados com insulinoterapia, recebendo administrações iniciais diárias de $0,25 \mathrm{U} / \mathrm{Kg}$ a cada doze horas, segundo protocolo adotado pela instituição. Seis cães com diagnóstico de DM foram monitorados individual e mensalmente por um período de doze meses, após o início do tratamento com insulina, até o controle glicêmico ou óbito, sendo três animais sobreviventes e três não sobreviventes. As coletas foram realizadas com o animal em jejum, pela venipunção da jugular, e o sangue foi acondicionado em tubos com EDTA e sem anticoagulante. A frutosamina e a glicose foram dosadas no soro; já a hemoglobina glicada foi dosada no sangue total. Os valores de glicose, hemoglobina glicada e frutosamina foram comparados com os valores obtidos de 100 cães hígidos ${ }^{(7)}$.

A análise da glicose foi realizada pelo método enzimático glicose oxidase no analisador semiautomático CELM SBA 200 (CELM, Barueri, Brasil), com kit comercial (Human do Brasil, Itabira, Brasil). A hemoglobina glicada foi determinada pelo método de resina de troca iônica com kit comercial (Human do Brasil) e a frutosamina pelo método cinético por redução do azul de nitrotetrazólio (NBT), com kit comercial (Labtest, Lagoa Santa, Brasil). A determinação da porcentagem da hemoglobina glicada e da frutosamina foram feitas por leitura em espectrofotômetro Quick-Lab (Dreake, São José do Rio Preto, Brasil).

Os resultados de glicose, hemoglobina glicada e frutosamina dos cães clinicamente sem alterações e dos cães com diabetes foram avaliados pelo teste de ANOVA e as médias comparadas pelo teste de Tukey, considerando-se $\mathrm{p}<0,05$.

Os resultados de glicose, hemoglobina glicada e frutosamina observados em cães com diabetes sob insulinoterapia foram avaliados para pesquisa de correlação, por meio do Teste de Correlação de Spearman. Ainda, as médias destas três variáveis foram avaliadas em três cães sobreviventes e três 
cães não sobreviventes sob tratamento com insulina, comparando-se os momentos de início, dois momentos intermediários e final da avaliação (óbito ou controle da glicemia), também utilizando-se o teste de ANOVA e as médias comparadas pelo teste de Tukey, considerando-se $p<0,05$.

\section{Resultados e Discussão}

Os 19 animais com DM utilizados neste estudo obtiveram valores de hemoglobina glicada e frutosamina superiores aos cães sem alteração clínica (Tabela 1). As dosagens de hemoglobina glicada e frutosamina são ferramentas muito empregadas porque fornecem informações dos níveis médios glicêmicos a curto e longo prazo $^{(8)}$, sendo de uso rotineiro na medicina humana, pois propiciam diagnóstico preciso de diabetes e monitoramento mais eficiente de pacientes em tratamento.

Uma vez que os animais utilizados eram provenientes da casuística de atendimento do hospital veterinário, sete cães não puderam ser monitorados durante a adaptação do protocolo de tratamento porque os proprietários não retornaram com os animais ao atendimento veterinário. Somente três cães que sobreviveram puderam ser acompanhados até o retorno de glicose, hemoglobina glicada e frutosamina aos valores de referência. Para efeitos de comparação, utilizaram-se três cães não sobreviventes que foram acompanhados até a última análise antes do óbito, para equiparar os grupos.

Tabela 1: Médias e desvios-padrão de glicose sérica, hemoglobina glicada e frutosamina de cães normais e cães com DM (ANOVA e Tukey, p<0,05)

\begin{tabular}{lcc}
\hline Avaliações & Cães normais & Cães com DM \\
\hline Glicose (mg/dL) & $95,1 \pm 11,9 \mathrm{a}$ & $494,2 \pm 180,03 \mathrm{~b}$ \\
HB glicada (\%) & $6,2 \pm 0,85 \mathrm{a}$ & $10,3 \pm 2,48 \mathrm{~b}$ \\
Frutosamina $(\mu$ mol/L) & $327,9 \pm 54,39 \mathrm{a}$ & $687,0 \pm 186,94 \mathrm{~b}$ \\
\hline
\end{tabular}

Letras iguais na mesma linha indicam médias sem diferença estatística significativa $(\mathrm{p}<0,05)$.

Houve correlação positiva entre glicose, hemoglobina glicada e frutosamina. A mais forte correlação observada ocorreu entre a glicose e a hemoglobina glicada; a análise entre a hemoglobina glicada e frutosamina resultou em correlação moderada (Tabela 2). Esta correlação comprova que a utilização destas avaliações para diagnóstico e monitoramento de cães com DM tem relevância na prática clínica. A moderada correlação verificada entre a hemoglobina glicada e a frutosamina pode ser explicada porque as duas relacionam-se diretamente com a glicemia sérica e, assim, indiretamente entre si. A glicemia sérica é afetada por diversos fatores, como, por exemplo, variação diurna, exercício, estresse, alimentação e medicação, o que não ocorre com a hemoglobina glicada e a frutosamina ${ }^{(9)}$, aumentando a margem de confiança para afirmar que o cão está com glicemia elevada e por tempo prolongado $^{(10)}$. Ambas as substâncias são geradas pela interação com a glicose plasmática ${ }^{(11,12)}$. Foi possível notar que a correlação mais elevada ocorreu entre a glicose e a hemoglobina glicada, resultado este observado também na comparação das avaliações entre animais sobreviventes e não sobreviventes, pois foram as únicas que mostraram resultados menores no grupo sobrevivente (Tabela 3). Infelizmente, a quantidade de animais avaliados durante o tratamento foi pequena, uma vez que a maioria não retornou para acompanhamento do tratamento instituído e ajuste da dose de insulina. Além disso, considerando-se as grandes diferenças encontradas entre os animais dos mesmos grupos, foi difícil demonstrar diferença estatística em todas as análises. Observou-se que, na última avaliação dos cães não sobreviventes, a média de frutosamina encontrada foi de $718 \mu \mathrm{mol} / \mathrm{L}$, considerada como controle glicêmico inadequado por Herring et al. ${ }^{(13)}$, que indicou um valor limite de $614 \mu \mathrm{mol} / \mathrm{L}$ para tratamento moderadamente adequado.

Loste et al. ${ }^{(14)}$ avaliaram as concentrações de frutosamina, em cães com diabetes e cães com insulinoma, pelo método cinético de redução do azul de nitrotetrazólio (NBT) e encontraram médias de 454,85 $\mu \mathrm{mol} / \mathrm{L}$ para cães diabéticos e $202,8 \mu \mathrm{mol} / \mathrm{L}$ para cães com insulinoma. Este valor obtido para cães com DM é inferior ao encontrado neste estudo, com média de $687 \mu \mathrm{mol} / \mathrm{L}$. Os estudos apontaram valores distintos, provavelmente pela gravidade do quadro de diabetes dos cães deste estudo, em que a maioria evoluiu ao óbito, o que consequentemente elevou os resultados da frutosamina. 
Tabela 2: Coeficientes de correlação entre glicose, hemoglobina glicada e frutosamina em cães com DM (Teste de Correlação de Spearman)

\begin{tabular}{lccc}
\multicolumn{1}{c}{ Matriz de correlação } & Glicose $(\mathbf{m g} / \mathbf{d L})$ & HB glicada $(\%)$ & Frutosamina $(\mu m o / L)$ \\
\hline Glicose $(\mathrm{mg} / \mathrm{dL})$ & 1 & 0,72477361 & 0,702394677 \\
HB glicada $(\%)$ & 0,72477361 & 1 & 0,675183174 \\
Frutosamina $(\mu \mathrm{mol} / \mathrm{L})$ & 0,702394677 & 0,675183174 & 1 \\
\hline
\end{tabular}

Tabela 3: Comparação da glicose, hemoglobina glicada e frutosamina entre cães sobreviventes e não sobreviventes, do início do tratamento até o fim do experimento (ANOVA e Tukey, $\mathrm{p}<0,05$ )

\begin{tabular}{|c|c|c|c|c|c|c|}
\hline & \multicolumn{2}{|c|}{ Glicose } & \multicolumn{2}{|c|}{ Hemoglobina glicada } & \multicolumn{2}{|c|}{ Frutosamina } \\
\hline & & Não & & Não & & Não \\
\hline & $\begin{array}{l}\text { Sobreviventes } \\
\qquad(\mathrm{n}=3)\end{array}$ & $\begin{array}{l}\text { sobreviventes } \\
\qquad(\mathrm{n}=3)\end{array}$ & $\begin{array}{l}\text { Sobreviventes } \\
\qquad(\mathrm{n}=3)\end{array}$ & $\begin{array}{c}\text { sobreviventes } \\
(\mathrm{n}=3)\end{array}$ & $\begin{array}{c}\text { Sobreviventes } \\
(\mathrm{n}=3)\end{array}$ & $\begin{array}{l}\text { sobreviventes } \\
\qquad(\mathrm{n}=3)\end{array}$ \\
\hline 1 & $478,5 \pm 14,84^{\mathrm{Aa}}$ & $427,33 \pm 22,5^{\mathrm{Aa}}$ & $9,75 \pm 0,35^{\mathrm{Aa}}$ & $8,70 \pm 0,72^{\mathrm{Aa}}$ & $707 \pm 66,46^{\mathrm{Aa}}$ & $556,33 \pm 69,8^{\mathrm{Aa}}$ \\
\hline 2 & $125,5 \pm 127,89^{\mathrm{Ba}}$ & $351,67 \pm 218,3^{\mathrm{Aa}}$ & $7,15 \pm 2,19^{\mathrm{Aa}}$ & $7,77 \pm 0,32^{\mathrm{Aa}}$ & $598 \pm 285,6^{\mathrm{Aa}}$ & $559,67 \pm 124,78^{\mathrm{Aa}}$ \\
\hline 3 & $160 \pm 2,82^{\mathrm{Ba}}$ & $342,00 \pm 171,1^{\mathrm{Aa}}$ & $6,7 \pm 0,14 \mathrm{Aa}$ & $8,55 \pm 1,41^{\mathrm{Aa}}$ & $359,5 \pm 6,36^{\mathrm{Aa}}$ & $511,00 \pm 213,5^{\mathrm{Aa}}$ \\
\hline 4 & $126 \pm 9,89 \mathrm{Ba}$ & $590,67 \pm 55,5^{\mathrm{Ab}}$ & $6,7 \pm 0,28^{\mathrm{Aa}}$ & $9,13 \pm 0,8^{\mathrm{Ab}}$ & $355 \pm 7,07^{\mathrm{Aa}}$ & $718,00 \pm 186,3^{\mathrm{Aa}}$ \\
\hline
\end{tabular}

Sako et al. ${ }^{(15)}$ observaram que valores de frutosamina acima de $300 \mu \mathrm{mol} / \mathrm{L}$ estão associados a controle glicêmico inadequado pelo tratamento com insulina. Como observado neste estudo, todos os animais que vieram à óbito apresentavam dosagem de frutosamina muito acima deste valor. No entanto, o intervalo encontrado em 100 cães não diabéticos para a frutosamina foi de 273,51 - 382,29 $\mu \mathrm{mol} / \mathrm{L}, \mathrm{o}$ que engloba o valor descrito por Sako et al. ${ }^{(15)}$ como limite para se avaliar a eficácia de um tratamento. Acredita-se que esta diferença se deva à metodologia empregada por aqueles pesquisadores, os quais utilizaram um método enzimático (FOD-TOOS). Davison et al.(16) encontraram valores de frutosamina em cães não diabéticos abaixo de $300 \mu \mathrm{mol} / \mathrm{L}$, e descrevem que cães com tratamento insulínico adequado possuem valores inferiores a $400 \mu \mathrm{mol} / \mathrm{L}$. Nos três cães em que foi possível fazer o monitoramente completo do tratamento, houve redução média da frutosamina a $355 \mu \mathrm{mol} / \mathrm{L}$, o que corrobora os resultados de Davison et al. ${ }^{(16)}$.

Wood E Smith ${ }^{(17)}$ relataram valores de hemoglobina glicada de 4,97\% para cães não diabéticos e 9,63\% para cães diabéticos, pela técnica de cromatografia em coluna, valores estes que foram semelhantes aos encontrados neste estudo, com resultados médios de hemoglobina glicada de $6,2 \%$ para cães não diabéticos e 10,3\% para cães com DM.

A DM apresenta uma complicação grave em cães, que é a cetoacidose diabética, caracterizada por alterações metabólicas extremas como hiperglicemia, acidose metabólica, acetonemia, desidratação e perda de eletrólitos ${ }^{(18)}$. Concentrações de hemoglobina glicada persistentemente acima dos níveis normais estão associadas a risco maior de complicações crônicas ${ }^{(19)}$. Este aumento da hemoglobina glicada foi observado nos cães deste estudo que apresentaram cetoacidose e que evoluíram a óbito, o que demonstra que esta enzima é mais precisa na detecção de complicações potencialmente fatais da DM.

\section{Conclusão}

A avaliação inicial de cães com DM deste estudo demonstrou valores médios de glicose de 494,2 md/ dL, hemoglobina glicada de $10,3 \%$ e frutosamina de $687 \mu \mathrm{mol} / \mathrm{L}$ antes do tratamento com insulina. Altos valores de hemoglobina glicada e a frutosamina relacionam-se com agravamento causado pelas complicações da DM, relacionados diretamente com mau prognóstico, devendo ser utilizadas tanto para diagnóstico como monitoramento da condição clínica do paciente. 


\section{Referências}

1. Little RR, Rohlfing CL, Wiedmeyer H. The national glycohemoglobin standardization program: a five-year progress report. Clinical Chemistry. 2005;47:1985-1992.

2. Sacks DB. Traslating hemoglobin A1C into average blood glucose: implications for clinical chemistry. Clinical Chemistry. 2008;54:1756-175. DOI: 10.1373/clinchem.2008.113282

3. Kaneko JJ. Carbohydrate metabolism and its disease. In: Kaneko JJ, Harvey JW, Bruss ML. Clinical Biochemistry of Domestic Animals. San Diego:Mosby Elsevier, 2008, p.62-70. English.

4. Hoening M. Comparative aspects of diabetes mellitus in dog and cats. Molecular and Cellular Endocrinology. 2002;197:221-229. DOI. 10.1016/S0303-7207(02)00264-2

5. Rooney NJ, Morant S, Guest C. Investigation into the Value of Trained Glycaemia Alert Dogs to Clients with Type I Diabetes. Plos One. 2015;8:1-12. Doi: 10.1371/journal.pone.0069921

6. Maggiore AD, Nelson RW, Dennis J, Johnson E, Kass PH. Efficacy of Protamine Zinc Recombinant Human Insulin for Controlling Hyperglycemia in Dogs with Diabetes Mellitus. Journal of the Veterinary Internal Medicine. 2012;26:109-115. DOI. 10.1111/j.1939-1676.2011.00861.x

7. Beltrame OC, Locatelli Dittrich R, Medeiros NC, Koch MO, Patricio LFL, Castilhos BQ, Pavelski M, Laskoski LM. Valores de Referência da hemoglobin glicada e frutosamina em cães. Semina: Ciências Agrárias. 2014;35:1865-1869. DOI. 10.5433/1679-0359.2014v35n4p1865

8. Nathan DM, Kuenen J, Borg R, Zheng H, Schoenfeld D, Heine RJ. Translating the A1C assay into estimated average glucose values. Diabetes Care. 2008;31:1473-1478. DOI:10.2337/dc08-0545

9. Jensen AL, AAES H. Reference interval and critical diference for canine serum fructosamine concentration. Veterinary Research Communication. 1992;16:317-325.

10. Sako T, Mori A, Lee P, Sato T, Mizutani H, Takahashi T, Kiyosawa Y, Tazaki H, Arai T. Serum glycated albumin: potential use as na index of glycemic control in diabetic dogs. Vet Res Comm. 2009;33:473-479. DOI:10.1007/s11259-008-9193-0

11. Bry L, Chen PC, Sacks DB. Effects of hemoglobin variants and chemically modified derivates on assay for glycohemoglobin. Chinical Chemistry. 2001;47:153-63.

12. Fracassi F, Corradini S, Hafner M, Boretti FS, Sieber-Ruckstuhl NS, Reusch CE. Detemir insulin for the treatment of diabetes mellitus in dogs. Journal of the American Veterinary Medical Association. 2015; 247:7378. Doi. 10.2460/javma.247.1.73.

13. Herring IP, Panciera DL, Werre SR. Longitudinal Prevalence of Hypertension, Proteinuria, and Retinopathy in Dogs with Spontaneous Diabetes Mellitus. Journal of Veterinary Internal Medicine. 2014;24:488-495. DOI. $\underline{10.1111 / j v i m .12286}$

14. Loste A, Marca MC, Perez M, Unzueta A. Clinical value of fructosamine measurements in non-healthy dogs. Veterinary Research Communications. 2001;.25:109-115.

15. Sako T, Mori A, Lee P, Takahashi T, Izawa T, Karasawa S, Furuuchi M, Azakami D, Mizukoshi M, Mizutani H, Kiyosawa Y, Arai T. Diagnostic significance of serum glycated albumin in diabetic dogs. Journal of Veterinary Diagnostic Investigation. 2008;20:634-638. DOI: 10.1177/104063870802000517

16. Davison LJ, Herrtage ME, Catchpole B. Study of 253 dogs in the United Kingdom with diabetes mellitus. Veterinary Record. 2013;156:467-471. DOI:10.1136/vr.156.15.467

17. Wood PA, Smith JE. Elevation rate of glycosylated hemoglobin in dogs after induction of experimental Diabetes Mellitus. Metabolism. 1982; 31(9):906-909.

18. Kitabchi AE, Umpierrez GE, Murphy MB, Barreth EJ, Kreisberg RA, Malone JI, Wall BM. Management of hyperglycemia crises in patients with diabetes. Diabetes Care. 2001;24(1):131-153. DOI: 10.2337/ diacare.24.1.131

19. Marca MC, Loste A, Unzueta A, Pérez M. Blood glycated hemoglobin evaluation in sick dogs. Canadian Journal of Veterianry Research. 2000;64:141-144. 